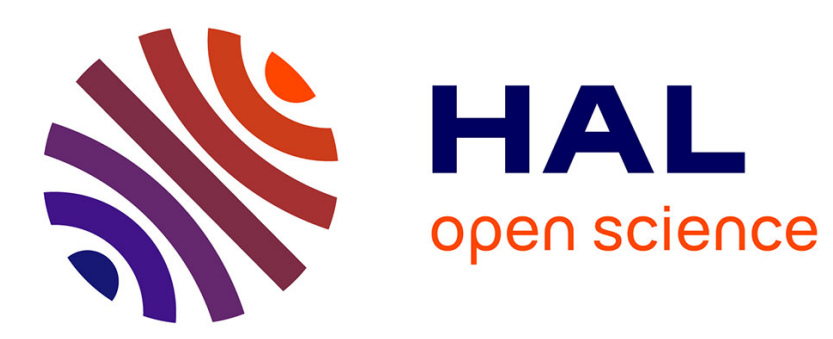

\title{
On the passivity based control of irreversible processes: a port-Hamiltonian approach.
}

Héctor Ramirez, Yann Gorrec, Bernhard Maschke, Françoise Couenne

\section{To cite this version:}

Héctor Ramirez, Yann Gorrec, Bernhard Maschke, Françoise Couenne. On the passivity based control of irreversible processes: a port-Hamiltonian approach.. Automatica, 2016, 64 (1), pp.105 - 111. 10.1016/j.automatica.2015.07.002 . hal-01303486

\section{HAL Id: hal-01303486 \\ https://hal.science/hal-01303486}

Submitted on 18 Apr 2016

HAL is a multi-disciplinary open access archive for the deposit and dissemination of scientific research documents, whether they are published or not. The documents may come from teaching and research institutions in France or abroad, or from public or private research centers.
L'archive ouverte pluridisciplinaire HAL, est destinée au dépôt et à la diffusion de documents scientifiques de niveau recherche, publiés ou non, émanant des établissements d'enseignement et de recherche français ou étrangers, des laboratoires publics ou privés. 


\title{
On the passivity based control of irreversible processes: a port-Hamiltonian approach
}

\author{
Hector Ramirez ${ }^{\mathrm{a}}$, Yann Le Gorrec ${ }^{\mathrm{a}}$, Bernhard Maschke $^{\mathrm{b}}$, Françoise Couenne $^{\mathrm{b}}$ \\ ${ }^{a}$ FEMTO-ST, UMR CNRS 6174, AS2M department, Université de Bourgogne Franche-Comté, Université de Franche-Comté, ENSMM, 24 rue Savary, F-25000 \\ Besanon, France. (e-mail: \{ramirez,legorrec\}@femto-st.fr) \\ ${ }^{b}$ LAGEP, UMR CNRS 5007, Université de Lyon, Université Lyon 1, Faculté Sciences et Technologie, 43 Boulevard du 11 Novembre 1918, F-69622 Villeurbanne, \\ France (e-mail:\{couenne,maschke\}@lagep.univ-lyon1.fr)
}

\begin{abstract}
Irreversible port-Hamiltonian systems (IPHS) have recently been proposed for the modelling of irreversible thermodynamic systems. On the other hand, a classical result on the use of the second law of Thermodynamics for the stabilization of irreversible processes is the celebrated thermodynamic availability function. These frameworks are combined to propose a class of Passivity Based Controller (PBC) for irreversible processes. An alternative formulation of the availability function in terms of internal energy is proposed. Using IPHS a matching-condition, which is interpreted in terms of energy-shaping, is derived and a specific solution that permits to assign a desired closed-loop structure and entropy rate is proposed. The approach can be compared with Interconnection and Damping Assignment - PBC, this method however leads in general to thermodynamically non-coherent closedloop systems. In this paper a system theoretic approach is employed to derive a constructive method for the control design. The closed-loop system is in IPHS form, hence it can be identified with a thermodynamic system and the control parameters related with thermodynamic variables, such as the reaction rates in the case of chemical reactions. A generic non-linear non-isothermal continuous stirred tank reactor is used to illustrate the approach.
\end{abstract}

Keywords: Passivity based control, port-Hamiltonian systems, irreversible thermodynamics, CSTR.

\section{Introduction}

The different suggestions for the modelling of irreversible thermodynamic processes as (dissipative) port-Hamiltonian systems (PHS) (Maschke \& van der Schaft, 1992; van der Schaft \& Maschke, 1995; Duindam et al., 2009) have led to a class of system called quasi-PHS (Hangos et al., 2001; OteroMuras et al., 2008; Eberard et al., 2007; Ramirez. et al., 2009; Dörfler et al., 2009; Hoang et al., 2011). These systems retain much of the dissipative port Hamiltonian structure, but differ by their structure (interconnection and dissipation) matrices and input vector fields which depend explicitly on the gradient of the Hamiltonian. This framework has recently been combined with the framework of the thermodynamic availability function (Alonso \& Ydstie, 1996; Ydstie \& Alonso, 1997; Alonso \& Ydstie, 2001) to derive Lyapunov conditions for the stabilization of irreversible thermodynamic systems (Ydstie, 2002; Hoang et al., 2011, 2012). From a control design perspective this implies that when looking for closed-loop potentials, for instance when passivity based control (PBC) techniques are applied (Ortega et al., 2001, 2002), the integrability conditions lead to partial differential equations which are nonlinear instead of linear. Furthermore, it is well known that for this case a physically consistent parametrization of the control problem is far from obvious (Kotyczka, 2013). This implies closed-loop systems without physical interpretation or very complex matching equations to solve during the design.

In this paper we shall consider the control of a class of such extensions of PHS, named Irreversible Port-Hamiltonian Sys- tems (IPHS) (Ramirez et al., 2013b,c). These systems embed by construction simultaneously the first (conservation of energy) and the second principle (irreversible creation of entropy). An incremental energy function, defined as an energy based availability function, is used as desired closed-loop Hamiltonian following Ramirez et al. (2013a, 2014). A Lyapunov condition is then derived and interpreted in terms of energy-shaping passivity based control (PBC) (Ortega et al., 2001, 2002). The Lyapunov condition is then further developed and a specific nonlinear solution, which permits to assign a desired closed-loop interconnection structure and entropy dissipation rate, is proposed.

The proposed design procedure consists in finding appropriate structure matrices and desired thermodynamic control functions to solve algebraically (Nunna et al., 2015; Acosta et al., 2008) the associated matching equations. The IPHS formulation allows to systematically parametrize the problem to derive the conditions for a globally stabilizing controller which preserves the IPHS structure in closed-loop. Since the structure of the closed-loop system is IPHS, it can be interpreted as a thermodynamic system and the parameters of the controller related with thermodynamic variables, such as the reaction rates in the case of chemical reactions.

The paper is organized as follows: Section 2 recalls the definition and physical interpretation of IPHS. In Section 3 the framework of the thermodynamic availability function is presented and a general Lyapunov condition is derived. Section 4 presents the main results of this paper. In Section 5 the ap- 
proach is applied to the example of a generic non-linear nonisothermal CSTR model. Finally Section 6 gives some closing remarks and comments on future work.

\section{Irreversible Port-Hamiltonian Systems}

Irreversible Port Hamiltonian Systems (IPHS) have been defined in Ramirez et al. (2013b) as an extension of Port Hamiltonian systems for the purpose of representing not only the energy balance but also the entropy balance, essential in thermodynamic systems.

Definition 1. (Ramirez et al., 2013b) An input affine IPHS is defined by the dynamic equation and output relation

$$
\begin{aligned}
& \dot{x}=R\left(x, \frac{\partial U}{\partial x}, \frac{\partial S}{\partial x}\right) J \frac{\partial U}{\partial x}(x)+g\left(x, \frac{\partial U}{\partial x}\right) u, \\
& y=g^{\top}\left(x, \frac{\partial U}{\partial x}\right) \frac{\partial U}{\partial x}(x)
\end{aligned}
$$

where $x(t) \in \mathbb{R}^{n}$ is the state vector, the smooth functions $U(x): \mathbb{R}^{n} \rightarrow \mathbb{R}$ and $S(x): \mathbb{R}^{n} \rightarrow \mathbb{R}$ represent, respectively, the internal energy (the Hamiltonian) and the entropy functions, $J \in \mathbb{R}^{n \times n}$ is a constant skew-symmetric structure (interconnection) matrix of the Poisson bracket (Maschke et al., 1992) acting on any two smooth functions $Z$ and $G$ as:

$$
\{Z, G\}_{J}=\frac{\partial Z^{\top}}{\partial x}(x) J \frac{\partial G}{\partial x}(x) .
$$

The real function $R=R\left(x, \frac{\partial U}{\partial x}, \frac{\partial S}{\partial x}\right)$ is composed by the product of a positive definite function $\gamma$ and the Poisson bracket between the entropy and the energy functions:

$$
R\left(x, \frac{\partial U}{\partial x}, \frac{\partial S}{\partial x}\right)=\gamma\left(x, \frac{\partial U}{\partial x}\right)\{S, U\}_{J},
$$

with $\gamma\left(x, \frac{\partial U}{\partial x}\right): \mathbb{R}^{n} \rightarrow \mathbb{R}, \gamma \geq 0$, a non-linear positive function. The input map is defined by $g\left(x, \frac{\partial U}{\partial x}\right) \in \mathbb{R}^{n \times m}$ with the input $u(t) \in \mathbb{R}^{m}$ a time dependent function.

The drift dynamic in (1) is defined by a non-linear relation between the time derivative $\dot{x}$ of the state variables and $\frac{\partial U}{\partial x}$, characterized by the modulating function $R\left(x, \frac{\partial U}{\partial x}, \frac{\partial S}{\partial x}\right)$, which explicitly depends on the differential of the energy $\frac{\partial U}{\partial x}$. The balance equations of the total energy and entropy functions of IPHS express the first and second principles of irreversible Thermodynamics: the conservation of energy and the irreversible creation of entropy due to irreversible phenomena. By skew-symmetry of $J$, the balance equation of the internal energy,

$$
\frac{d U}{d t}=y^{\top} u
$$

expresses that the system (1) is a lossless dissipative systems with (energy) supply rate $y^{\top} u$ (Willems, 1972). The balance equation of the entropy function is given by

$$
\begin{aligned}
\frac{d S}{d t} & =R\left(x, \frac{\partial U}{\partial x}, \frac{\partial S}{\partial x}\right) \frac{\partial S^{\top}}{\partial x} J(x) \frac{\partial U}{\partial x}+\frac{\partial S^{\top}}{\partial x} g\left(x, \frac{\partial U}{\partial x}\right) u \\
& =\gamma\left(x, \frac{\partial U}{\partial x}\right)\{S, U\}_{J}^{2}+\left(g^{\top}\left(x, \frac{\partial U}{\partial x}\right) \frac{\partial S}{\partial x}\right)^{\top} u .
\end{aligned}
$$

By Definition 1 the first term is positive: $\gamma\left(x, \frac{\partial U}{\partial x}\right)\{S, U\}_{J}^{2}=$ $\sigma\left(x, \frac{\partial U}{\partial x}\right) \geq 0$. For irreversible thermodynamic systems, this term represents the internal entropy production and its positivity expresses the second principle of Thermodynamics. The second term in (4) corresponds to the definition of an entropy supply rate. For further details on IPHS and its thermodynamic interpretation we refer the reader to Ramirez et al. (2013b).

\section{Energy shaping of IPHS}

In Ramirez et al. (2013a) the framework of the thermodynamic availability function, formalized for the control of thermodynamic systems by Alonso \& Ydstie (2001) and with roots in the works of Keenan (1951) and Willems (1972), has been used to derive a Lyapunov condition for the stability analysis of IPHS. Using the convexity of the internal energy function, a convex extension named energy based availability function has been defined and shown to be a Lyapunov function candidate for the closed-loop system. This has been done following Alonso \& Ydstie (2001); Ydstie (2002); Hoang et al. (2011, 2012), where an entropy based availability function is constructed for irreversible thermodynamic systems.

In the sequel the stability condition presented in Ramirez et al. (2013a) is developed and it is shown that it defines an energy shaping controller (Ortega et al., 2001, 2002) with respect to a new closed-loop Hamiltonian and supply rate.

Definition 2. The energy based availability function is defined as

$$
A\left(x, x^{*}\right)=U(x)+U_{a}\left(x, x^{*}\right)
$$

with

$$
U_{a}\left(x, x^{*}\right)=-U\left(x^{*}\right)-\frac{\partial U}{\partial x}\left(x^{*}\right)^{\top}\left(x-x^{*}\right)
$$

and $x^{*}$ an equilibrium point.

Assumption 3. The availability function $A\left(x, x^{*}\right)$ is strictly positive with minimum $A\left(x=x^{*}\right)=0$ where $x^{*}$ is an equilibrium point.

This assumption is fulfilled for any equilibrium point of a monophasic thermodynamic systems if one of the extensive variables is fixed since then the internal energy is a strictly convex function (Jillson and Ydstie, 2007).

It is clear from Definition 2 that the energy based availability function qualifies as a Lyapunov function candidate for controlled IPHS. Define an availability-conjugated output defining an availability supply rate of the availability function,

$$
\tilde{y}=g^{\top}\left(\frac{\partial U}{\partial x}(x)-\frac{\partial U}{\partial x}\left(x^{*}\right)\right)=g^{\top} \frac{\partial A}{\partial x}\left(x, x^{*}\right)
$$

Proposition 4. Let $x^{*}$ be an equilibrium point for (1) and $A\left(x, x^{*}\right)$ satisfy Assumption 3 with $u=\beta\left(x, x^{*}\right)$ satisfying

$$
\gamma\{S, U\}_{J}\{A, U\}_{J}+\tilde{y}^{\top} \beta=-s,
$$

with $s\left(x, x^{*}\right)>0, \forall x \neq x^{*}$ and $s\left(x, x^{*}\right)=0$ for $x=x^{*}$. Then $x^{*}$ is globally asymptotically stable. 
Proof. $A$ is by Definition 2 a Lyapunov function candidate for (1). The time derivative of $A$ along the trajectories of (1) is given by

$$
\begin{aligned}
\frac{d A}{d t}= & \left(\frac{\partial U}{\partial x}(x)-\frac{\partial U}{\partial x}\left(x^{*}\right)\right)^{\top} \frac{d x}{d t} \\
= & R\left(\frac{\partial U}{\partial x}(x)-\frac{\partial U}{\partial x}\left(x^{*}\right)\right)^{\top} J \frac{\partial U}{\partial x}(x)+ \\
& \left(\frac{\partial U}{\partial x}(x)-\frac{\partial U}{\partial x}\left(x^{*}\right)\right)^{\top} g \beta
\end{aligned}
$$

and using (8) we finally obtain

$$
\frac{d A}{d t}=-s
$$

Since $A$ is strictly positive $x^{*}$ is an isolated minimum, and since $s$ only vanishes at $x^{*}$ asymptotic stability follows invoking La Salle's invariance Theorem on a region around $x^{*}$.

Proposition 4 defines an energy shaping controller (Ortega et al., 1998). Indeed, assume there exists a function $U_{a}\left(x, x^{*}\right)$, which represents the added/removed energy by the controller, such that the closed-loop energy function (availability function) can be written as $A=U(x)+U_{a}\left(x, x^{*}\right)$. Then from (3), the existence of $U_{a}$ such that (10) is satisfied implies the existence of a state feedback $u=\beta\left(x, x^{*}\right)$ such that

$$
\beta\left(x, x^{*}\right)^{\top} y(x)=-\dot{U}_{a}\left(x, x^{*}\right)-s(x) .
$$

The closed-loop energy function $A$ is defined by construction, hence by combining (6) and (11) we obtain

$$
\beta\left(x, x^{*}\right)^{\top} y(x)=\frac{\partial U}{\partial x}\left(x^{*}\right)^{\top} \dot{x}-s(x),
$$

and using (1) gives the matching equation (8). Hence, Proposition 4 defines an energy shaping controller with added/removed energy $U_{a}$.

\section{Interconnection and entropy rate assignment}

In this section we shall further elaborate on a class of control which not only assigns the energy based availability function as closed-loop Hamiltonian but also shapes the structure of the system by assigning a desired interconnection matrix and closed-loop entropy rate. Consider the following target system

$$
\dot{x}=-\mathcal{M}\left(x, \frac{\partial S}{\partial x}, \frac{\partial A}{\partial x}\right) \frac{\partial A}{\partial x}(x),
$$

with $\mathcal{M}\left(x, \frac{\partial S}{\partial x}, \frac{\partial A}{\partial x}\right) \geq 0$. The time variation of $A$ is

$$
\dot{A}=-\frac{\partial A^{\top}}{\partial x}(x) \mathcal{M}\left(x, \frac{\partial S}{\partial x}, \frac{\partial A}{\partial x}\right) \frac{\partial A}{\partial x}(x) \leq 0
$$

which implies that under some additional properness conditions (12) is asymptotically stable.

The target system (12) can be interpreted within the framework of IDA-PBC (Ortega et al., 2001, 2002) if $\mathcal{M}=$
$J_{d}(x)-M(x)$, with $M(x)$ a symmetric positive semi-definite matrix defining a pseudo-Riemannian metric expressed by the so called Ginzburg-Landau (dissipative) bracket (Grmela \& Öttinger, 1997), defined in some local coordinates for two smooth functions $Z$ and $G$ as,

$$
[Z, G]_{M}=\frac{\partial Z^{\top}}{\partial x}(x) M(x) \frac{\partial G}{\partial x}(x) .
$$

In this case (12) is a PHS. The drawback of selecting a PHS as target system for the control of IPHS is that it leads to non-linear matching (partial differential) equations. This motivates the algebraic approach employed in this paper, where the closed-loop Lyapunov function is fixed $a$ priori as the availability function using the thermodynamic properties of IPHS. Some example of use of algebraic approaches for solving matching equations in PBC design can be found for instance in Acosta et al. (2008); Nunna et al. (2015).

\subsection{A locally asymptotically stabilizing controller}

We shall now elaborate on the matching equation presented in the previous section and derive the conditions for shaping the Hamiltonian and the structure of the system. Define the control input as $u=\gamma\{S, U\}_{J} \tilde{u}$, with $\tilde{u}$ an auxiliary input, then the matching equation (8) becomes

$$
\gamma\{S, U\}_{J}\left(\{A, U\}_{J}+\tilde{y}^{\top} \tilde{u}\right)=-s .
$$

where $s$ is the rate of decrease of the availability function defined in (10). Notice that (14) may be compared to the stabilization condition for reversible Hamiltonian systems. In that case $\gamma\{S, U\}_{J}=1$ and the condition $\{A, U\}_{J}+\tilde{y}^{\top} \tilde{u}=-s$ expresses the decrease of the shaped closed-loop Hamiltonian function. For IPHS, from (14), we see that this condition is modulated by the nonlinear term $R=\gamma\{S, U\}_{J}$, which is not signed defined since the bracket $\{S, U\}_{J}$ may take positive or negative values. Let us consider a particular solution to (14) in the following form

$$
\tilde{y}^{\top} \tilde{u}=-\{A, U\}_{J}-\{S, U\}_{J}[A, A]_{M}+\{A, A\}_{J_{d}}
$$

where $J_{d}(x)=-J_{d}^{\top}(x)$ is a desired closed-loop interconnection matrix defining a Poisson bracket. Notice that the bracket $\{A, A\}_{J_{d}}=0$, expressing the conservation of the closed-loop energy with respect to $J_{d}$. This is referred to as interconnection assignment. Substituting (15) in (14), we have that the dissipation function is given by

$$
s=\gamma\{S, U\}_{J}^{2}[A, A]_{M}
$$

Using the definition of $\tilde{y}$ (Eq. (7)) and noting that (15) is multiplied from the left by $\frac{\partial A}{\partial x}^{\top}$, the control law $\tilde{u}$ satisfying (15) and being independent of the co-energy variables has to satisfy

$$
g \tilde{u}=\left(-J \frac{\partial U}{\partial x}-\{S, U\}_{J} M \frac{\partial A}{\partial x}+J_{d} \frac{\partial A}{\partial x}\right) .
$$


The linear least square solution with respect to the Euclidean norm of this equation considering $\tilde{u}=\beta(x)$ is given by,

$$
\begin{aligned}
& \beta(x)= \\
& \begin{aligned}
g^{\dagger}(x)\left(-\{S, U\}_{J} M(x)+J_{d}(x)\right)( & \left.\frac{\partial U}{\partial x}(x)-\frac{\partial U}{\partial x}\left(x^{*}\right)\right) \\
& -g^{\dagger}(x) J \frac{\partial U}{\partial x}(x),
\end{aligned}
\end{aligned}
$$

which exists if and only if the following matching equation is satisfied (Campbell \& Meyer, 2009):

$$
\begin{aligned}
-g^{\perp}(x)\{S, U\}_{J} M(x) & \left(\frac{\partial U}{\partial x}(x)-\frac{\partial U}{\partial x}\left(x^{*}\right)\right) \\
+g^{\perp}(x) & \left(\left(J_{d}-J\right) \frac{\partial U}{\partial x}(x)-J_{d} \frac{\partial U}{\partial x}\left(x^{*}\right)\right)=0
\end{aligned}
$$

where $g^{\dagger}(x)=\left[g^{\top}(x) g(x)\right]^{-1} g^{\top}(x)$ is the Moore-Penrose pseudo inverse and $g^{\perp}(x)$ a left full rank annihilator of $g(x)$, i.e., $g^{\perp}(x) g(x)=0$. Equations (17) and (18) may be interpreted in terms of IDA-PBC. Indeed, take again the case reversible case, $\gamma\{S, U\}_{J}=1$, then $u=\tilde{u}$ and it is observed that the solution corresponds to the IDA-PBC solution for the PHS

$$
\dot{x}=J \frac{\partial U}{\partial x}+g \tilde{u}
$$

with target system given by

$$
\dot{x}=\left(-M(x)+J_{d}(x)\right) \frac{\partial A}{\partial x} .
$$

In the present case, since $\gamma\{S, U\}_{J} \neq 1$, the target system of the equivalent IDA-PBC problem is given by

$$
\dot{x}=\left(-\{S, U\}_{J} M(x)+J_{d}(x)\right) \frac{\partial A}{\partial x},
$$

where the matrix $\{S, U\}_{J} M(x)$ is not a positive (semi) definite since $\{S, U\}_{J}$ is of arbitrary sign. This "contradiction" comes of course from the fact that the previous interpretation has been performed with respect to the auxiliary input $\tilde{u}$ and not the actual control input $u=\gamma\{S, U\}_{J} \tilde{u}$. Nevertheless, it also expresses the irreversible nature of the controlled system: The bracket $\{S, U\}_{J}$, which for irreversible thermodynamic systems corresponds to the physical driving force, appears explicitly in the structure matrix of IPHS making the control design nonlinear with respect to the states and co-states $\left(\frac{\partial U}{\partial x}\right)$.

The result is summarized in the following Proposition.

Proposition 5. Let $x^{*}$ be an equilibrium point for (1). Assume there exist matrices $M(x) \geq 0$ and $J_{d}(x)=-J_{d}^{\top}(x)$, and a fullrank left annihilator $g^{\perp}(x)$ of $g(x)$ satisfying (18). Then $u=$ $\gamma\{S, U\}_{J} \beta$ with $\beta$ as in (17) locally asymptotically stabilizes $x^{*}$. Furthermore, the closed-loop dynamic is

$$
\dot{x}=\left(-\sigma M+R J_{d}\right) \frac{\partial A}{\partial x} .
$$

with $\sigma=\gamma\{S, U\}_{J}^{2} \geq 0$.
Proof. Equation (21) is obtained by replacing the control law $u=\gamma\{S, U\}_{J} \beta$ in (1) under the assumption that (18) is satisfied. Since $M(x)$ is positive semi-definite, the asymptotic stability follows by applying La Salle's invariance principle in a region around the equilibrium. The region of attraction is given by the largest subset that excludes $\{S, U\}_{J}=0$, which corresponds to the thermodynamic equilibrium, and which is normally excluded from the region of operation of the system.

The closed-loop system resembles an IPHS with respect to the energy based availability function, but the modulating function $R=\gamma\{S, U\}_{J}$ is defined with respect to the open-loop structure matrix $J$ and Hamiltonian $U(x)$. The dissipation of the closed-loop system is given by (16), and may be equivalently written in term of the internal entropy production or entropy rate $\sigma$ as $s=\sigma[A, A]_{M}$, hence it is directly related with the irreversible thermodynamic dissipation of the system.

The matching equation (18) is an algebraic condition since the closed-Hamiltonian (and hence also $U_{a}$ ) has been fixed. The design parameters are the dissipation matrix $M$, the desired interconnection matrix $J_{d}$ and up to some degree $g^{\perp}$. These matrices should be chosen such that (18) is satisfied and can be used to assign the desired closed-loop dynamic. For some cases, it is possible to use relations arising from the physical properties of the system to solve (18). A particular case is when the interconnection matrix is not changed, i.e., $J_{d}=J$, and only some closed-loop dissipation is added.

Corollary 6. Assume $J_{d}=J$, then $x^{*}$ is locally asymptotically stable if

$$
\begin{aligned}
g^{\perp} J \frac{\partial U}{\partial x}\left(x^{*}\right) & =0, \quad \text { and } \\
g^{\perp} M & =0 .
\end{aligned}
$$

Furthermore, the control defines a purely energy-balancing + entropy injecting controller.

The thermodynamic equilibrium is normally excluded from the region of operation of the system, however it should be noticed that $\{S, U\}_{J}=0$ implies $\frac{d A}{d t}=0$. The equilibrium is hence only locally asymptotically stable, and any equilibrium too-close to the thermodynamic equilibrium could present convergence problems.

\subsection{A globally asymptotically stabilizing controller}

In order to overcome the limitations of the previous design we now fix as control objective to render the closed-loop system IPHS and assign a desired closed-loop entropy rate. This, from a physical point of view, is equivalent to change the thermodynamic equilibrium in closed-loop. Consider the following solution to (8)

$$
\tilde{y}^{\top} u=-R\{A, U\}_{J}-\sigma_{d}[A, A]_{M}+R_{d}\{A, A\}_{J_{d}}
$$

where $R_{d}=\gamma_{d}\{S, A\}_{J_{d}}$ and $\sigma_{d}=\gamma_{d}\{S, A\}_{J_{d}}^{2}$ with $\gamma_{d}>0$ a non-vanishing positive function. These two functions which are defined by the controllers design parameters $A, J_{d}$ and 
$\gamma_{d}$, are respectively, a designed non-linear modulating function and a closed-loop entropy rate. $R_{d}$ and $\sigma_{d}$ only vanish at $\frac{\partial A}{\partial x}\left(x^{*}\right)=0$, which corresponds to the desired closed-loop equilibrium. The difference with (15), where an auxiliary input is used to factor-out the bracket $\{S, A\}_{J}$, in (24) new modulating functions, $\sigma_{d}$ and $R_{d}$, are introduced as design parameter to introduce a closed-loop thermodynamic equilibrium that coincides with the desired equilibrium $x^{*}$.

The control law $u$ has to satisfy

$$
g u=\left(-R J \frac{\partial U}{\partial x}-\sigma_{d} M \frac{\partial A}{\partial x}+R_{d} J_{d} \frac{\partial A}{\partial x}\right),
$$

And the linear least square solution with respect to the Euclidean norm is given by

$$
\begin{aligned}
\beta(x)=g^{\dagger}(x)\left(R_{d} J_{d}-\sigma_{d} M\right)\left(\frac{\partial U}{\partial x}(x)\right. & \left.-\frac{\partial U}{\partial x}\left(x^{*}\right)\right) \\
& -g^{\dagger}(x) R J \frac{\partial U}{\partial x}(x),
\end{aligned}
$$

which exists if and only if

$$
\begin{aligned}
g^{\perp}(x)\left(R_{d} J_{d}-\sigma_{d} M\right)\left(\frac{\partial U}{\partial x}(x)-\right. & \left.\frac{\partial U}{\partial x}\left(x^{*}\right)\right) \\
& -g^{\perp}(x) R J \frac{\partial U}{\partial x}(x)=0
\end{aligned}
$$

The result is summarized in the following proposition.

Proposition 7. Let $x^{*}$ be an equilibrium point for (1). Assume there exist matrices $M(x) \geq 0$ and $J_{d}(x)=-J_{d}^{\top}(x)$, a scalar functions $\gamma_{d}>0$ such that $\sigma_{d}=\gamma_{d}\{S, A\}_{J_{d}}^{2}$ and $R_{d}=\gamma_{d}\{S, A\}_{J_{d}}$, and a full-rank left annihilator $g^{\perp}(x)$ of $g(x)$ satisfying (26). Then $u=\beta$ with $\beta$ as in (25) globally asymptotically stabilizes $x^{*}$. Furthermore, the closed-loop dynamic is

$$
\dot{x}=\left(-\sigma_{d} M+R_{d} J_{d}\right) \frac{\partial A}{\partial x} .
$$

Proof. The proof is analogous to the proof of Proposition 5. The global asymptotic stability follows by applying La Salle's invariance principle in a region around $x^{*}$ noting that $\frac{d A}{d t}$ only vanishes at $x^{*}$.

The closed-loop system is an IPHS with dissipation function $s=\sigma_{d} \frac{\partial A}{\partial x}^{\top} M \frac{\partial A}{\partial x}$. Indeed, we see from (27) that if $M=0$, then (27) is an IPHS with respect to $A$ according to Definition 1.

It's important to remark that (27) can be derived using IDA-PBC, however finding an appropriate physically consistent parametrization is far from obvious when dealing with nonmechanical or electro-mechanical systems (Kotyczka, 2013). The systematic control design approach employed in the derivation of Proposition 5 led to a thermodynamic consistent parametrization of the control parameters $A, J_{d}, M$, and $\sigma_{d}$ in Proposition 7. This result can be regarded as a thermodynamic equivalent of IDA-PBC. Indeed (27) is again an IPHS with structure matrix $R_{d} J_{d}$ and energy function $A$ (interconnection assignment and energy shaping), and the dissipation assignment given by matrix $\sigma_{d} M$ assures the convergence to the minimum of the closed-loop energy function.

Corollary 6 can be generalised for this case as follows.
Corollary 8. $x^{*}$ is globally asymptotically stable if

$$
\begin{array}{rlrl}
g^{\perp} J & =0, & \text { and } \\
g^{\perp} J_{d} & =0, \quad \text { and } \\
g^{\perp} M & =0 . &
\end{array}
$$

Condition (30) is in general easy to fulfil since the only restrictions on $M$ are that it should be positive semi-definite and symmetric. Condition (29) is only required if $J_{d}$ is chosen different to the null matrix. On the other hand, condition (28) is more restrictive, since $J$ and $g$ (and up to an important degree also $g^{\perp}$ ) are defined by the structure of the system. Nevertheless, since $J$ expresses the conserved quantities of the system we may find this condition automatically fulfilled for systems whose input maps are related with the physical invariants. In that case selecting $J=J_{d}$ reduces (28) and (29) to one equivalent condition. This point will be illustrated on the example of the CSTR.

Remark 9. No assumption has been made on the stability of the open-loop equilibrium. The control is designed using a state feedback derived from Lyapunov arguments, allowing to deal with multiple or/and unstable open-loop equilibria like in the case of exothermic chemical reaction systems.

\section{Example: the CSTR}

Let us consider for simplicity and without restricting to a single reaction in a continuous stirred tank reactor (CSTR) with the following reversible reaction scheme

$$
\sum_{i=1}^{m} \zeta_{i} B_{i} \stackrel{r}{\rightleftharpoons} \sum_{i=1}^{m} \eta_{i} B_{i}
$$

with $\zeta_{i}, \eta_{i}$ being the constant stoichiometric coefficients for species $B_{i}$ in the reaction. We shall consider that the CSTR satisfies the following standard operation assumptions (Aris, 1989; Favache \& Dochain, 2009)

Assumption 10. 1) The reactor operates in liquid phase, 2) The molar volumes of each species are identical and the total volume, denoted by $V$, in the reactor is maintained constant, 3) The initial number of moles of a species in the reactor is equal to the number of moles of the inlet of the same species, 4) For a given steady state temperature $T$ and steady state input there is only one possible steady state for the mass (numbers of moles) balance.

Remark 11. The last point of Assumption 10 is standard and doesn't imply that the chemical reaction system doesn't admit multiple stable or unstable equilibrium points. It refers to that each steady state temperature is associated to one unique steady state temperature. 


\subsection{IPHS model (Ramirez et al., 2013b)}

The CSTR has been extensively studied using IPHS and contact geometry in Ramirez et al. (2013b) and the reader is referred to that work for a detailed deduction of the IPHS model. The dynamic equation of the CSTR may be expressed as

$$
\dot{x}=R J \frac{\partial U}{\partial x}(x)+g\left(x, \frac{\partial U}{\partial x}\right) u
$$

with state vector $x=\left[\mathbf{n}^{\top}, S\right]^{\top}$, where $\mathbf{n}=\left(n_{1}, \ldots, n_{m}\right)^{\top}$ with $n_{i}$ the number of moles of the species $i$ inside the reactor, $S$ the total entropy, the internal energy $U(x)$ as Hamiltonian function,

$$
J=\left[\begin{array}{cccc}
0 & \ldots & 0 & \bar{v}_{1} \\
0 & \ldots & 0 & \vdots \\
0 & \ldots & 0 & \bar{v}_{m} \\
-\bar{v}_{1} & \ldots & -\bar{v}_{m} & 0
\end{array}\right], \quad \frac{\partial U}{\partial x}=\left[\begin{array}{c}
\mu_{1} \\
\vdots \\
\mu_{m} \\
T
\end{array}\right],
$$

where the structure matrix $J$ is a constant skew-symmetric matrix whose elements are the signed stoichiometric coefficients of the chemical reaction $\bar{v}_{i}=\zeta_{i}-\eta_{i}$, which is positive or negative depending on whether the species $i$ is a product or a reactant, the differential $\frac{\partial U}{\partial x}$ is the vector of intensive variables, where $T$ and $\mu_{i}$ are respectively the temperature in the reactor and the chemical potential of the species $i$. The modulating function $R$ is given by

$$
R=\gamma\left(x, \frac{\partial U}{\partial x}\right)\{S, U\}_{J}=\left(\frac{r V}{T \mathcal{A}}\right) \mathcal{A}
$$

with $\gamma=\frac{r V}{T \mathcal{A}}$ and $\{S, U\}_{J}=\mathcal{A}$, where $r(\mathbf{n}, T)$ is the reaction rate which depends on the temperature $T$ and on the reactant mole number vector $\mathbf{n}$ (Horn \& Jackson, 1972; Feinberg, 1987), $\mathcal{A}=-\sum_{i=1}^{m} \bar{v}_{i} \mu_{i}$ is the chemical affinity of the reaction and corresponds to the thermodynamic driving force of the chemical reaction. The input vector is $u=\left[u_{1}, u_{2}\right]^{\top}$ with $u_{1}=F / V$ the dilution rate, where $F$ is the volumetric flow rate, and $u_{2}=Q$ the heat flux from the cooling jacket. The input map is given by

$$
g=\left[\begin{array}{cc}
\tilde{\mathbf{n}} & \mathbf{0} \\
\phi(x) & \frac{1}{T}
\end{array}\right]
$$

with $\tilde{\mathbf{n}}=\mathbf{n}_{\mathbf{e}}-\mathbf{n}$, where $\mathbf{n}_{\mathbf{e}}=\left(n_{e 1}, \ldots, n_{e m}\right)^{\top}$ is the vector containing the number of moles of species $i$ at the inlet and $\phi(x)=\sum_{i=1}^{m}\left(n_{e i} s_{e i}-n_{i} s_{i}\right)+\frac{n_{e i}}{T}\left(h_{e i}-T s_{e i}-\mu_{i}\right)$, where $s_{e i}, s_{i}$ and $h_{e i}$ are respectively the inlet molar entropy, the molar entropy and the inlet specific molar enthalpy of species $i$.

The assumption of constant total volume imposes a constraint over the total outlet flow (Couenne et al., 2006, 2008), i.e. in the present case the total outlet flow is chosen equal to the total inlet flow. We underline that the assumption of constant volume guarantees that the energy based availability function $A$ is strictly convex.

\subsection{Stabilization of the CSTR}

A constructive method to compute globally asymptotically stabilizing solutions to the stability condition is provided by Proposition 7. Let us select $J_{d}=J$ such that Corollary 8 can be used. First we shall verify that (28) is satisfied and then we shall look for a matrix $M$ such that (30) holds. The first step is to find a full-rank left annihilator for the input map. A possible choice is the $(m-1) \times n$ matrix

$$
g^{\perp}=\left[\begin{array}{ccccccc}
\tilde{n}_{2} & -\tilde{n}_{1} & 0 & \ldots & 0 & 0 & 0 \\
0 & \tilde{n}_{3} & -\tilde{n}_{2} & \ldots & 0 & 0 & 0 \\
0 & 0 & \ddots & \ddots & \ddots & 0 & 0 \\
0 & 0 & \ldots & 0 & \tilde{n}_{m} & -\tilde{n}_{m-1} & 0
\end{array}\right] .
$$

Using this annihilator we compute from (22)

$$
g^{\perp} J=\left[\begin{array}{cccc}
0 & \ldots & 0 & \bar{v}_{1} \tilde{n}_{2}-\bar{v}_{2} \tilde{n}_{1} \\
0 & \ldots & 0 & \bar{v}_{2} \tilde{n}_{3}-\bar{v}_{3} \tilde{n}_{2} \\
\vdots & \vdots & \vdots & \vdots \\
0 & \ldots & 0 & \bar{v}_{m-1} \tilde{n}_{m}-\bar{v}_{m} \tilde{n}_{m-1}
\end{array}\right]
$$

and it remains to verify that $g^{\perp} J=0$, which is true if

$$
\frac{\tilde{n}_{1}}{\bar{v}_{1}}=\frac{\tilde{n}_{2}}{\bar{v}_{2}}=\cdots=\frac{\tilde{n}_{m-1}}{\bar{v}_{m-1}}=\frac{\tilde{n}_{m}}{\bar{v}_{m}} .
$$

It has been shown (Prigogine \& Defay, 1954) for batch reactors that (32) is actually the expression of De Donder's extent of reaction

$$
\frac{n_{0_{i}}-n_{i}}{\bar{v}_{i}}=\xi
$$

This property can easily be extended to the CSTR case as soon as Assumption 10 is satisfied, i.e., when the initial number of moles of each species equals the number of moles at the inlet: $\mathbf{n}(t=0)=\mathbf{n}_{0}=\mathbf{n}_{e}$ (see also Aris (1989)). Hence (28) is automatically fulfilled. This comes from the fact that $J$ expresses the stoichiometry of the reaction and $g$ the mole (mass) balance relation. Since the reactor operates at constant volume, the total mass becomes an invariant for the reaction, and $g^{\perp} J=0$ is simply the mathematical expression of this invariant.

Condition (30) can be solved by noting that the last column of $g^{\perp}$ is zero. This implies that any matrix $M(x)=M^{\top}(x) \geq 0$ for which the first $m$ rows and columns forms a null submatrix is solution to (30). A (simple) possible choice is $M=$ $\operatorname{diag}(0, \ldots, 0,1)$, which corresponds to a diagonal matrix with all elements equal to zero except the last element of its diagonal which is 1 . The closed-loop system then takes the form

$$
\dot{x}=\left(-\sigma_{d} M+R_{d} J\right) \frac{\partial A}{\partial x} .
$$

The closed-loop system is an IPHS with dissipation, with the availability function as closed-loop Hamiltonian. Moreover, if no damping injection is considered $(M=0)$, then the controller corresponds to an energy-shaping controller. The dissipation is given by $-\sigma_{d} M \frac{\partial A}{\partial x}$, it is modulated by the desired entropy production $\sigma_{d}$ and acts only on the entropy balance. This is consistent with the thermodynamic interpretation of the closedloop system. The closed-loop entropy balance is given by

$$
\dot{S}=-\gamma_{d} \sum_{i=1}^{m} v_{i}\left(\mu_{i}(x)-\mu_{i}\left(x^{*}\right)\right)-\sigma_{d}\left(T-T^{*}\right) .
$$


From this balance equation we see how the first term contributes to the energy-balancing and the second term to the entropy injection. The time derivative of $A$ is

$$
\frac{d A}{d t}=-\sigma_{d}\left(T-T^{*}\right)^{2},=-\gamma_{d}\left(\mathcal{A}-\mathcal{A}^{*}\right)^{2}\left(T-T^{*}\right)^{2} .
$$

The decrease of the energy based availability function is related to the desired irreversible entropy rate, which is positive definite and only zero at the desired equilibrium. In order to complete the stability proof, we apply La Salle's invariance Theorem in a sufficiently small region around $T=T^{*}$. From Assumption 10 we have that there is only one equilibrium for each temperature and that $T=T^{*} \Leftrightarrow \mathcal{A}=\mathcal{A}^{*}$, hence we can conclude that the closed-loop systems is globally asymptotically stable.

The tuning parameter in the proposed controller is the scalar function $\gamma_{d}>0$. From (33) and (27) we observe that this function can be used to increase/decrease and shape the closed-loop dissipation and the closed-loop interconnection matrix. Recall that in open-loop $\gamma=\frac{r V}{T \mathcal{A}}$, where the the most important function is the reaction rate $r$, which characterises the chemical reaction. Hence a thermodynamically consistent choice for the controller is $\gamma_{d}=r_{d}(x) \frac{V}{T^{*} \mathcal{A}^{*}}$, with $r_{d}(x)$ a desired reaction profile. A simple choice that guarantees $\sigma_{d}>0$ is $r_{d}=r^{*}$ which is the open-loop reaction rate evaluated at the desired equilibrium.

\section{Conclusion}

This paper presents a constructive PBC method based on IPHS formulation and the thermodynamic availability function. The controller has been interpreted in terms of energy-shaping PBC and further developed to derive a specific non-linear solution, which permits to assign a desired closed-loop interconnection structure and entropy rate. The matching condition is an algebraic condition whose solution is parametrized by the desired closed-loop interconnection and damping matrices. It is shown that a physically (thermodynamically) coherent closedloop system can not be in PHS form, and hence standard PBC techniques such as IDA-PBC fail in the choice of the ideal target system. The proposed controller globally stabilizes the closed-loop system an renders it IPHS with dissipation. This allows to interpret the closed-loop system as a thermodynamic system and relate the control parameters with thermodynamic variables, such as the reaction rates in the case of chemical reactions.

The example of a generic non-linear non-isothermal CSTR has been used to illustrate the approach. For this case the solution to the matching equation follows directly from the IPHS model. Future work will deal with numerical implementations of the controller under realistic operation conditions.

\section{Acknowledgements}

The authors would like to thank the editors and the reviewers for their constructive comments and suggestions. This work was supported by French sponsored projects HAMECMOPSYS and Labex ACTION under reference codes ANR-11BS03-0002 and ANR-11-LABX-0001-01, respectively.

\section{References}

Acosta, J., Ortega, R., Astolfi, A., \& Sarras, I. (2008). A constructive solution for stabilization via immersion and invariance: The cart and pendulum system. Automatica, 44, $2352-2357$.

Alonso, A. A., \& Ydstie, B. E. (1996). Process systems, passivity and the second law of thermodynamics. Computers and Chemical Engineering, 20, 1119-1124.

Alonso, A. A., \& Ydstie, B. E. (2001). Stabilization of distributed systems using irreversible thermodynamics. Automatica, 37, 1739-1755.

Aris, R. (1989). Elementary chemical reactor analysis. Chemical Engineering. Stoneham, USA: Butterworths.

Campbell, S. L., \& Meyer, C. D. (2009). Generalized Inverses of Linear Transformations. Society for Industrial and Applied Mathematics.

Couenne, F., Jallut, C., Maschke, B., Breedveld, P., \& Tayakout, M. (2006). Bond graph modelling for chemical reactors. Mathematical and Computer Modelling of Dynamical Systems, 12, 159-174.

Couenne, F., Jallut, C., Maschke, B., Tayakout, M., \& Breedveld, P. (2008). Structured modeling for processes: A thermodynamical network theory. Computers $\mathcal{E}$ Chemical Engineering, 32, 1120 - 1134.

Dörfler, F., Johnsen, J., \& Allgöwer, F. (2009). An introduction to interconnection and damping assignment passivity-based control in process engineering. Journal of Process Control, 19, 1413-1426.

Duindam, V., Macchelli, A., Stramigioli, S., \& Bruyninckx, H. (Eds.) (2009). Modeling and Control of Complex Physical Systems - The Port-Hamiltonian Approach. Berlin, Germany: Springer-Verlag.

Eberard, D., Maschke, B. M., \& van der Schaft, A. J. (2007). An extension of Hamiltonian systems to the thermodynamic phase space: Towards a geometry of nonreversible processes. Reports on Mathematical Physics, 60, 175-198.

Favache, A., \& Dochain, D. (2009). Thermodynamics and chemical systems stability: The CSTR case study revisited. Journal of Process Control, 19, 371-379.

Feinberg, M. (1987). Chemical reaction network structure and the stability of complex isothermal reactorsi. the deficiency zero and deficiency one theorems. Chemical Engineering Science, 42, 2229 - 2268.

Grmela, M., \& Öttinger, H. (1997). Dynamics and thermodynamics of complex fluids. i. development of a general formalism. Physical Review E, 56, 6620 6632.

Hangos, K. M., Bokor, J., \& Szederkényi, G. (2001). Hamiltonian view on process systems. AIChE Journal, 47, 1819-1831.

Hoang, H., Couenne, F., Jallut, C., \& Le Gorrec, Y. (2011). The port Hamiltonian approach to modelling and control of continuous stirred tank reactors. Journal of Process Control, 21, 1449-1458.

Hoang, H., Couenne, F., Jallut, C., \& Le Gorrec, Y. (2012). Lyapunov-based control of non isothermal continuous stirred tank reactors using irreversible thermodynamics. Journal of Process Control, 22, 412-422.

Horn, F., \& Jackson, R. (1972). General mass action kinetics. Archive for Rational Mechanics and Analysis, 47, 81-116.

Keenan, J. H. (1951). Availability and irreversibility in Thermodynamics. British Journal of Applied Physics, 2, 183.

Kotyczka, P. (2013). Local linear dynamics assignment in IDA-PBC. Automatica, 49, 1037 - 1044 .

Maschke, B., \& van der Schaft, A. (1992). Port controlled Hamiltonian systems: modeling origins and system theoretic properties. In Proceedings of the 3rd IFAC Symposium on Nonlinear Control Systems, NOLCOS'92 (pp. 282-288). Bordeaux, France.

Maschke, B., van der Schaft, A., \& Breedveld, P. (1992). An intrinsic Hamiltonian formulation of network dynamics: Non-standard Poisson structures and gyrators. Journal of the Franklin Institute, 329, 923-966.

Nunna, K., Sassano, M., \& Astolfi, A. (2015). Constructive interconnection and damping assignment for port-controlled hamiltonian systems. Automatic Control, IEEE Transactions on, PP, 1-1.

Ortega, R., Loria, A., Nicklasson, P., \& Sira-Ramirez, H. (1998). Passivitybased control of Euler-Lagrange Systems. Communications and Control Series. Berlin: Springer.

Ortega, R., van der Schaft, A., Mareels, I., \& Maschke, B. (2001). Putting energy back in control. IEEE Control Systems Magazine, 21, 18- 32.

Ortega, R., van der Schaft, A., Maschke, B., \& Escobar, G. (2002). Interconnection and damping assignment passivity based control of port-controlled Hamiltonian systems. Automatica, 38, 585-596. 
Otero-Muras, I., Szederkényi, G., Alonso, A. A., \& Hangos, K. M. (2008). Local dissipative Hamiltonian description of reversible reaction networks. Systems $\mathcal{E}$ Control Letters, 57, 554-560.

Prigogine, I., \& Defay, R. (1954). Chemical Thermodynamics volume 1 of Treatise on Thermodynamics. London, Great Britain: Longmans Green and Co.

Ramirez, H., Gorrec, Y. L., Maschke, B., \& Couenne, F. (2013a). Passivity based control of irreversible port Hamiltonian systems. In Proceedings of the 2013 IFAC Workshop on Thermodynamic Foundations of Mathematical Systems Theory (TFMST'13). Lyon, France.

Ramirez, H., Le Gorrec, Y., \& Maschke, B. (2014). Interconnection and damping assignment - passivity based control of irreversible port Hamiltonian systems. In Proceedings of the 19th IFAC World Congress. Cape Town, South Africa.

Ramirez, H., Maschke, B., \& Sbarbaro, D. (2013b). Irreversible portHamiltonian systems: A general formulation of irreversible processes with application to the CSTR. Chemical Engineering Science, 89, 223 - 234.

Ramirez, H., Maschke, B., \& Sbarbaro, D. (2013c). Modelling and control of multi-energy systems: An irreversible port-Hamiltonian approach. European Journal of Control, 19, 513 - 520 .

Ramirez., H., Sbarbaro, D., \& Ortega, R. (2009). On the control of non-linear processes: An IDA-PBC approach. Journal of Process Control, 19, 405 414

van der Schaft, A., \& Maschke, B. (1995). The Hamiltonian formulation of energy conserving physical systems with external ports. Archiv für Elektronik und Übertragungstechnik, 49, 362-371.

Willems, J. (1972). Dissipative dynamical systems part I: General theory. Archive for Rational Mechanics and Analysis, 45, 321-351.

Ydstie, B. E. (2002). Passivity based control via the second law. Computers and Chemical Engineering, 26, 1037-1048.

Ydstie, B. E., \& Alonso, A. A. (1997). Process systems and passivity via the clausius-planck inequality. Systems $\mathcal{E}$ Control Letters, 30, 253 - 264. 